nium acetate solution and five cc. acetic acid (thirty per cent.) and digest for half an hour at $70^{\circ} \mathrm{C}$., by which time the precipitation is complete.

Filter, washing five or six times with hot ammonium acetate solution (ten per cent.), stirring up the precipitate with the jet each time. Ignite with a low flame until the paper is charred, increase the heat, and, when the paper is completely consumed, blast for a minute. The precipitate is the normal aluminum phosphate and its weight multiplied by the factor $0.4 \mathrm{I} 8$ gives the $\mathrm{Al}_{2} \mathrm{O}_{3}$.

The iron oxide is determined volumetrically, preferably by the bichromate method, in a solution of the precipitate of iron oxide and calcium phosphate thrown down by the caustic potash. It is also determined separately, by the same method, in a solution of five grams of the rock in dilute hydrochloric acid (I-I).

My thanks are due to Mr. H. E. Cutts, A.M., for valuable assistance in the above investigation.

LABORATORY OF STILIWELI \& GLADDING.

New YoRk CiTy.

\title{
SOME THOUGHTS ABOUT LIQUIDS.
}

\section{BY CLARENCE L. SPEYERS.}

Received Tune 3, 1896.

ONSIDER an empty closed space. Imagine a quantity of $\checkmark$ liquid put into it, enough to fll the space with vapor and leave some liquid over. A portion of the liquid changes into vapor and passes into the previously empty space above the liquid and continues doing so until the pressure of the vapor reaches a certain value, when the vaporization ceases.

The usual way of explaining this vaporization starts out by assuming that, with the exception of the surface, the liquid is perfectly homogeneous in a physical sense. That is, there is not a single particle of the liquid which for any appreciable length of time is different from any other particle, but of course, spaces between the particles of liquid are recognized. At the surface of the liquid, however, a distinction is to be made. For outside the surface, the activities are different from those within the surface, otherwise there would be no boundary. So that the 
particles at the surface are subjected to activities that are different in different directions, and consequently the particles so situated will behave differently from those particles entirely within the liquid.

In van der Waal's theory the mutual attractions of the particles of the liquid are considered as the restraining force to keep the particles more or less together. This assumed force must be very great-a good many hundred atmospheres. Inside the liquid, below the surface, the attraction is equal in all directions, but at the surface it acts only in one direction, inwards, normal to the surface. Now, although the force restraining the particles of liquid from separating is so great, yet the theory of common acceptance assumes that some particles do break away from the mass of the liquid and form vapor. The liquid is said to evaporate. It is hard to accept this view of the case, particularly as electrical results point towards an exceedingly quiet condition of affairs within the body of liquids.

Still admitting that the particle does break away from this attraction, it cannot do so without an abundant supply of energy, which must be accounted for. It does not seem right to find this energy in the heat of vaporization, for a particle of liquid will voluntarily take heat energy from the liquid to do this work, and so go off as a particle of vapor at the sacrifice of the energy of the liquid.

It is not possible to prevent a liquid from vaporizing by refusing to give it heat; it will take the required heat from the rest of the liquid. In other words, the condition of the liquid state strongly favors vaporization.

The common theory tries to get over this difficulty by claiming that the particle which gets away, gets away by virtue of an inherent kinetic energy greater than the attractic energy of the particies of liquid, that is, greater than the force denoted by van der Waal by $K=\frac{a}{v^{2}}$, and that it possesses this excess of kinetic energy in the body of the liquid, before it got away, and that it got away only by virtue of this excess of kinetic energy. Similarly with all particles in the liquid having a kinetic energy 
greater than the energy of the mutual attraction of the particles the one for the other.

Still again admitting that the particles with the greater kinetic energy do succeed in getting out, we shall have to look for a loss in the mean kinetic energy of the liquid, to be shown by a fall in temperature. This is the case and in this respect theory and fact agree, for vaporization lowers the temperature of the liquid. In regard to the vapor, however, we meet with this difficulty. The kinetic energy of a given particle while within the liquid might be just sufficient to carry it beyond the sphere of action of the liquid, in which case the kinetic energy remaining to the particle after reaching freedom would be little and its temperature should be less than that of the liquid; on the other hand, the kinetic energy of the given particle might be far more than sufficient to carry it beyond the sphere of action of the liquid and then the kinetic energy that the particle still has after reaching freedom, should be more or less great. Consequently a particle of vapor, just after getting out of the liquid, might have any temperature between absolute zero and $\infty^{\circ}$, in some cases perhaps touching the inferior limit, but of course, never reaching the superior limit. As a consequence of this we should expect to find the temperature of the vapor very different from that of the liquid; but this is not the case, observation has never shown that the temperature of the vapor is very different from that of the liquid which produces it. However, this is not a fatal objection, for we can assume that the mean of kinetic energy, while within the liquid, of the particles that escape from the liquid is of just such a value that after they have all got out of the liquid, the diminution of their mean kinetic energy, due to the attraction of the liquid, brings the kinetic energy left to them to the mean kinetic energy of the liquid. This seems like a forced explanation, very forced, but still pethaps not more so than the theory it is intended to help.

However, we are not yet out of the difficulty. For what becomes of the kinetic energy lost by the particles as they pass out into the vapor state?

So far as the liquid is concerned, it may take the form of heat energy and so lessen the quantity required to supply the deficit 
due to the escape of the particles with great kinetic energy from the liquid. But all of the lost kinetic energy cannot be absorbed here in the liquid, some must also go into the vapor particles. It may take the form of heat, as we have suggested in the case of the liquir, but then we have to assume that the kinetic energy, while within the liquid, of the particles that escape is of just such a value that, after they have all got out of the liquid, the diminution in their mean kinetic energy, due to the attraction of the liquid plus this correction, brings the kinetic energy left to them to the mean kinetic energy of the liquid, which is absurd.

Nor does the attractive energy seem to be stored up as potential energy, as in the case of a stone raised above the surface of the earth, for there is no evidence at all that a vapor particle tends toward the liquid as the stone does toward the earth. When the particle gets out of the liquid it seems to be utterly indifferent to the liquid.

Of course the mutual attraction that all bodies have for each other is left out of account.

Nor is there any sign of electrical action, at least if the experiments made up to the present time are conclusive.

There are then a good many very serious objections to the present theory of vaporization.

First, in accounting for the escape of the vapor.

Second, in accounting for the temperature of the vapor.

Third, in accounting for the kinetic energy lost by the particle in getting through the surface of the liquid and beyond the sphere of action of the liquid particies.

Let us now turn our attention to another view of the case. Consider a liquid which has no vapor-tension of its own, a nonvolatile liquid, but which can dissolve gases. The liquid and gas are supposed to act according to Henry's law, that is, the ratio of concentration of the gas in the liquid part and in the gaseous part is to be constant, or in other words, the quantity of gas dissolved by the liquid is to be proportional to the pressure on the gas.

In such a system there are three constituents to be considered. The gas in the gaseous state, the gas in solution, and the solvent. 
The state of the dissolved gas is not positively known, but in all probability it is in a state corresponding to a gas under high pressure for these reasons. In the first place, it is hard to see how a substance like nitrogen, for example, could be in the liquid state in a solution of moderate concentration. Great pressure is required to liquefy it even when the temperature is far below the ordinary temperature, and at the ordinary temperature it has hitherto been found impossible to liquefy nitrogen, no matter how great the pressure. Still it would be consistent with the ordinarily accepted theory to claim that the attraction of the particles of solvent conld overcome the great internal energy of the gas particles and bind them down to a lesser activity and produce the liquid state. But on the other hand, modern investigation has very plainly shown that dissolved substances have a gaseous nature; the particles of the dissolved body are free to assert their physical individuality. That is to say, the solvent is to be considered rather as a medium through which the dissolved body can be put under certain conditions, the conditions varying to some extent with each solvent, but all solvents having the common action of allowing a sort of gasification of the substance dissolved, In general the solvent is not to be considered as a substance which unites with the dissolved body, forming a new compound. For example, consider anhydrous calcium chloride. When this is treated with water there is strong evidence of combination of the two to form calcium chloride hydrate. If the quantity of water is properly adjusted the whole of it combines with the calcium chloride, forming a crystallized hydrate. If this crystalline substance is treated with more water, solution begins and during this process, which is the real solution, there is no sign of chemical action. It is true, some scientists, particularly those of the English school, have denied this and have claimed to find strong evidence of a chemical action during the process of solution, but so far all such claims have turned out to be mere opinions based upon very doubtful measurements.

So we are to look upon solution as being a change in which the dissolved body is gasified. Sometimes a further change, electrolytic dissociation, takes place, but that is outside the 
scope of this article. It is in best accordance with what we know about other bodies to assume that the dissolved nitrogen is in the form of a gas, and to recognize two states in the solution, the gaseous state of the substance in solution and the liquid state of the solvent.

Let us now pass on to a liquid which gives off vapor. The purpose of this article is to justify the view that this vapor behaves toward the liquid just as the nitrogen did toward its solvent in the previous case, of course, with the obvious limitations due to identity in the composition of vapor and liquid.

The boundary dividing vapor from liquid is commonly supposed to be at the surface of the liquid, although the possibility of a differentiation occurring inside the liquid does not seem to be denied, for so far as could be found out by the writer, the question of such a possibility has never been raised.

The tendency for a liquid to vaporize and the pressure of its saturated vapor is evidently a function of temperature only. There seems to be no reason, therefore, why the fluid should not separate into vapor and liquid within the surface of the liquid. That it is possible for vapor to be there follows from what we know about the gaseous nature of the substances in solution. It is rather odd that this view of the case was not adopted at the outset by chemists.

According to this view, when we heat a liquid we increase the energy of translatory motion, we increase its temperature. But besides this we cause a separation of some of the liquid particles from the body of the liquid, bringing them into a state of freedom, such that they can behave just as the particles of any other substance would do in the same solvent. This of course will consume considerable energy. These free particles of vapor in the liquid we shall call dissolved vapor particles. So that on heating in liquid we produce dissolved vapor and raise the temperature of the whole fluid; possibly we do more, but at any rate we do these two things. Now by Clausius' theory of the true specific heat, the heat required to raise only the temperature of a unit mass of substance one degree, should be the same whatever the state of the substance may be, and the value of the true specific heat should be the value of the specific heat when 
the substance is in such a state that the heat added can only change its temperature and not do any other internal work, namely when the substance is in a state of gas. So if we subtract from the specific heat of the liquid the specific heat of the gas, the remainder should be the heat consumed in other internal work, and if no other internal work is done than the rise in temperature and production of dissolved vapor, we should get the heat required to change some of the liquid into dissolved vapor. The quantity changed into vapor however is so far unknown.

The dissolved vapor is supposed to be produced until its energy balances the energy of the liquid part.

Suppose, for example, we heat one gram of water one degree in a closed vessel which does not allow it to give off gaseous vapor. The heat required will be about one calorie, depending upon the initial temperature; one calorie is near enough for our purpose. A part of the heat goes to increase the translatory motion and is the true specific heat; but another part, perhaps the whole of the remainder, we claim goes to produce dissolved vapor. Subtracting the true specific heat of water, namely the specific heat of water vapor at a ligh temperature $=0.4776$, we have left 0.5224 as the heat required to change a certain unknown quantity of water into dissolved water vapor, provided that no internal work is done but the two kinds we have considered. We shall assume this to be true until there is evidence of a more complex change.

Now suppose a space be made over the liquid, to let a certain quantity, say one per cent., be changed into gaseous vapor. It is of course evident, if the theory be at all tenable, that the vapor arising from the liquid comes from the dissolved vapor and bears to the dissolved vapor the same relation that the nitrogen did to the dissolved nitrogen. Comparatively little heat should be required in this process, for most of the change has been effected in the body of the liquid. Whatever is required here should be looked upon as the true heat of vaporization; that which is usually so called we are to consider as incluring the heat required to change a unit mass of liquid into dissolved vapor as well as the heat required to vaporize the unit mass of dissolved 
vapor. The two quantities should evidently be kept carefully separated.

Let us now proceed to determine the concentration of the dissolved water vapor. As the dissolved water vapor is supposed to be like a dissolved gas, Henry's law should give us some aid in finding the quantity. We might assume, in the first place, that the relative vapor density of a liquid at two different temperatures gives the relative osmotic pressures of the dissolved vapor at those temperatures, were it not for the uncertainty as to how the temperature affects the pressure of the vapor and the osmotic pressure of the dissolved vapor. It would not do to assume that each was affected in the same degree by a change in temperature. But our theory does allow us to claim in the case of a given liquid at a constant temperature that two different vapor-tensions will correspond to two different concentrations of the dissolved vapor by Henry's law, and that the relative vapor-tensions are as the relative concentrations of the dissolved vapor. Now we can change at will, within quite a wide range, the vapor tension of a liquid without changing its temperature and without introducing any complications.

To understand this let us refer back to the original conception of the dissolved vapor. If we have liquid water in a vessel with any number of gases under moderate pressure, the partial pressure of the saturated water vapor will be very nearly the same as if it alone were present in the space containing the gases. So when we dissolve a substance in water it would seem as if we might argue that the osmotic pressure of the dissolved substance should not affect the pressure of the dissolved water vapor. However the conditions in the two cases are very different. In the first case there is abundant space for the water vapor so that all that is necessary is time for the concentration of the water vapor to reach the same value no matter how many gases may be present, provided of course that the total pressure be not very great. When however the total pressure is great, the vapor-tension of the liquid diminishes very much. This is just the condition that holds in a liquid. The volume available for a dissolved substance is very small, and so any thing put into this space will very materially lessen the space available for the dis- 
solved vapor, particularly as the quantities used in solutions are generally very much greater than those used in the gaseous state.

Suppose we have $n$ gram-molecules of a substance whose molecules do not dissociate on dissolving, say sugar, and dissolve it in water. Let $v$ be the number of gram-molecules of dissolved vapor after the $n$ gram-molecules of substance have been dissolved, then the total number of gram-molecules present in solution will be $v+n$, and the relative number of gram-molecules of substance dissolved to total number of gram-molecules in solution is

$$
\frac{n}{v+n} \text {. }
$$

Now let $j$ be the concentration of the dissolved vapor when alone in the liquid, and $j^{\prime}$ its concentration after the new substance has been added, in this case the sugar. $j-j^{\prime}$ will be the decrease in the concentration of the dissolved water vapor due the addition of the $n$ gram-molecules of sugar, and since a grammolecule of all substances occupies the same volume, the decrease in concentration $j-j^{\prime}$ will be the same whatever the substance dissolved may be, provided the same number of grammolecules be taken in each case, or the decrease in concentration of the dissolved vapor is proportional to the number of grammolecules dissolved in a certain fixed volume of solution. If the temperature is constant the concentration of the dissolved water vapor cannot rise above the value $j$, which it has when only dissolved vapor is present; when we try to get above this value the dissolved vapor turns to liquid water. Hence the number of gram-molecules in a unit volume must be fixed, if the temperature is constant, that is

$$
v+n=\text { constant. }
$$

We have, therefore,

$$
\frac{j-j^{\prime}}{j}=\alpha \frac{n}{v+n}
$$

where $\alpha$ is a constant.

$j-j^{\prime}$ can be calculated by van't Hoff's law, and $n$ is known, but the other quantities are not, so neither $j$ or $v$ can be calcula ted from this equation. 
There is however another relation that can be deduced.

The concentration of the dissolved vapor is measured by its osmotic pressure.

Let $\pi, \phi$, be respectively osmotic pressure and osmotic volume of the dissolved vapor, when it alone is present ; $\pi^{\prime}, \phi^{\prime}$, the corresponding quantities when a substance is in solution; $p, v$, the pressure and volume of the vapor in contact with the pure solvent; $p^{\prime}, v^{\prime}$, the corresponding quantities when a substance is in solution.

Consider an isothermal reversible cycle composed of the following parts.

By means of a semipermeable diaphragm let a gram-molecule of dissolved vapor pass from the pure solvent, the work will be

$$
-\pi \phi=-R T \text {. }
$$

Let the gram-molecule of vapor expand until it has a pressure of $\pi$ the work will be

$$
-\int_{\pi^{\prime}}^{\pi} \phi d \pi=-R T l \frac{\pi}{\pi^{\prime}}
$$

Let it then pass into the solution; the work will be

$$
+\pi^{\prime} \phi^{\prime}=+R T \text {. }
$$

Let $x$ gram-molecules pass out of the solution in the form of vapor; the work will be

$$
-x p^{\prime} v^{\prime}=-x R T
$$

where $x$ denotes the number of gram-molecules of gaseous vapor necessary to make one gran-molecule of dissolved vapor.

Let the $x$ gram-molecules of vapor be compressed until the pressure equals $p$; the work will be

$$
+x \int_{p^{\prime}}^{p} v d p=+x R T \frac{p}{p^{\prime}} .
$$

Let the $x$ gram-molecules be driven into the pure solvent; the work will be

$$
+x p v=+x R T .
$$


Thus the cycle is completed. The quantity of solution is supposed to be so large that the addition and removal of the quantity of the solvent used in the cycle will have no appreciable effect upon the concentration of the solution.

The sum of the changes of energy must be zero, so

$-R T-R T \frac{\pi}{\pi^{\prime}}+R T-x R T+x R T l \frac{p}{p}+x R T=0 ;$

Hence, $\quad \frac{\pi}{\pi^{\prime}}=\left(\frac{p}{p^{\prime}}\right)^{x}$

We shall assume that $x$ equals $\mathrm{I}$; there is no good reason for thinking otherwise, and the simplicity in the structure of dissolved bodies favors this assumption.

From the theory we have

$$
j_{j}=\frac{\pi}{\pi^{\prime}}
$$

We have therefore from I through 3 and 2 ,

$$
\mathrm{I}-\frac{p^{\prime}}{p}=\frac{p-p^{\prime}}{p}=\alpha \frac{n}{v+n}
$$

but from experiment.

$$
\frac{p-p^{\prime}}{p}=\frac{n}{N+n}
$$

where $N$ is the number of gram-molecules of liquid in which $n$ gram-molecules of substance have been dissolved.

Hence,

$$
\alpha \frac{n}{\nu+n}=\frac{n}{N+n}
$$

Now as equation (6) is true for any small value of $n$ it will be true for a value so small in comparison with $\nu$ and $N$, that it may be neglected, and so

$$
\frac{\alpha n}{\nu}=\frac{n}{N},
$$

or,

$$
\alpha=\frac{\nu}{N}
$$


Substituting in (6) we have

$$
\frac{v}{N} \frac{n}{v+n}=\frac{n}{N+n}
$$

or,

$$
v=N
$$

That is, the concentration of the dissolved vapor is the same as the concentration of the liquid, or in other words, all the solvent is to be considered as dissolved vapor.

This is very interesting, for it is in effect the same conclusion that van der Waals reached in his celebrated treatise, though he pursued a very different method.

It would seem from this result that matters were left in about the same state that they were in at the outset; that the view of dissolved vapor was no better than the old view, which claimed that the change into vapor took place only on the surface of the liquid. But we have really gained several things.

In the first place we have found that reasoning from the analogy that a dissolved gas and the same gas in contact with the solvent bears to the liquid and its vapor we got to the idea of dissolved vapor and from that to a result in agreement with a much older theory.

Secondly, we have found that a liquid is to be looked upon as a condensed gas, not simply condensed in the sense that it is a matter compressed into smaller space, but condensed in the sense that the gaseous activity, pressure, is carried into the liquid condition, and we are to treat a liquid as we would a gas.

Thirdly, it follows from this view that a substance dissolved is simply brought into the same condition that the liquid is in, and consequently should have the same property of exerting an osmotic pressure that the liquid has.

Finally, what causes the condensed gaseous state? Until this is answered the problem of liquid and gas is essentially unsolved. That it is due to an attraction between the molecules, is hardly possible, as we have seen at the beginning of this paper. Indeed so soon as we begin to reflect upon the complications that are introduced the moment the ideas of molecule and attraction are brought into an investigation, and these complications are all 
the time increasing instead of diminishing, the more natural and simple appears the view of Ostwald that we shall find a better solution of such problems in energy alone, matter being only a collection of energies in space.

Now as to the value of the osmotic pressure in some liquids.

In $1000 \mathrm{cc}$. of water there are

$$
\frac{\mathrm{IO0O}}{\mathrm{I} 8}=55.55 \mathrm{gram} \text {-molecules. }
$$

Every gram-molecule at $25^{\circ} \mathrm{C} .\left(=298^{\circ}\right.$ absolute temperature $)$ in $1000 \mathrm{cc}$. has a pressure of

$$
\frac{22222}{1000} \cdot \frac{298}{273} \cdot 0.76 \mathrm{~m}
$$

Hence for the $55.55 \mathrm{gram}-$ molecules of water we have

$$
\pi=\frac{22222}{1000} \cdot \frac{298}{273} \cdot 0.76 \frac{1000}{18}=1024 \text { meters of mercury. }
$$

In $1000 \mathrm{cc}$. methyl alcohol there are

$$
\frac{1000}{32} \cdot 0.79 \text { gram-molecules, }
$$

and hence for methyl alcohol we have

$$
\pi=\frac{22222}{1000} \cdot \frac{298}{273} \cdot 0.76 \frac{1000}{32} \cdot 0.79=455 \mathrm{~m} .
$$

In 1000 cc. ethyl alcohol there are

$$
\frac{\text { rooo }}{46} \cdot 0.79 \text { gram-molecules, }
$$

and hence for ethyl alcohol we have

$$
\pi=\frac{22222}{1000} \cdot \frac{298}{273} \cdot 0.76 \frac{1000}{46} \cdot 0.79=316 \mathrm{~m} .
$$

In Iooo cc. propyl alcohol there are

$$
\frac{1000}{60} \cdot 0.80 \text { gram-molecules, }
$$

and hence for propyl alcohol we have

$$
\pi=\frac{22222}{1000} \cdot \frac{298}{273} \cdot 0.76 \frac{1000}{46} \cdot 0.80=249 \mathrm{~m} .
$$


In $1000 \mathrm{cc}$. chloroform there are

$$
\frac{1000}{\text { II9 }} \text {. I.52 gram-molecules, }
$$

and hence for chloroform we have

$$
\pi=\frac{22222}{1000} \cdot \frac{298}{273} \cdot 0.76 \frac{1000}{\text { II }} \cdot 1 \cdot 52=235 \mathrm{~m} .
$$

In $1000 \mathrm{cc}$. toluene there are

$$
\frac{\text { IO00 }}{9^{2}} \cdot 0.88 \text { gram-molecules, }
$$

and hence for toluene we have

$$
\pi=\frac{22222}{1000} \cdot \frac{298}{273} \cdot 0.76 \frac{1000}{92} \cdot 0.88=176 \mathrm{~m} .
$$

Rutgers College.

[CONTRTBUTIONS FROM THE LABORATORIES OF THE School OF MiNing, KINGSTON, ONTARIO.]

\section{VOLUMETRIC ESTIMATION OF LEAD.}

BY FRED. J. POPE.

Received May 2r, 1896.

UITE frequently of late, the attention of readers of chemical journals has been directed to various methods ${ }^{1}$ for estimating lead volumetrically. But, while some of these methods are superior to any previously made public, yet, for none of them is that degree of accuracy claimed which is so essential in a reliable quantitative operation. The chief objection to all of these methods is the use of an outside indicator. However, by using an inside indicator and modifying slightly the usual preliminary steps (necessary for the conversion of the ore into the sulphate) results have been obtained by the writer which are quite satisfactory.

The operation may be briefly outlined as follows: The lead is first converted into lead sulphate, then into lead acetate. Excess of standard potassium bichromate is added, which precipitates the lead as lead chromate. The unused potassium bichromate is reduced by excess of standard arsenious acid, and this latter

1 This Journal, 17, 901; Engineering and Mining Journal, July 7, 1894. 